\title{
Specula Vaticana.
}

La nuova Specula Vaticana in Roma e collocata in parte nell' antica Specula Gregoriana fondata nel 1582 , in occasione della riforma del calendario, la quale sembrava opportunissima per osservazioni soprattutto di meteorologia, e in parte nell' ampia torre costrutta sotto Leone IV, posta in fondo al giardino Vaticano, acconcia specialmente alle osservazioni di astronomia.

Secondo il programma proposto, la nuova Specula si occuperà di indagini di meteorologia, di magnetismo terrestre, di geodinamica e di astronomia.

Per la meteorologia, le condizioni della località sceita sono le migliori che si possono avere non pure in Roma, ma anche altrove. La Specula sarà fornita di tutti gli strumenti richiesti non solo per le osservazioni dirette, ma eziandio per la registrazione continua dei diversi ele. menti meteorici, e vi si faranno altre osservazioni speciali, massime di fotografia meteorologica. Nè s'intralasceranno le ricerche d'elettricità atmosferica.

Per il magnetismo terrestre vi saranno tutti gli apparecchi per le variazioni a registrazione fotografica (i primi che s'introducano negli Osservatori italiani), e gli altri per le determinazioni assolute delle costanti del magnetismo terrestre.

Per la sismica si posseggono già parecchi pregiati istrumenti avuti in dono dai loro inventori o costruttori, tra $\mathrm{i}$ più celebrati d'Italia, e altri ne verranno in seguito.

Per la parte astronomica, siccome esistono già in Roma tre Osservatori astronomici, cosi per non moltiplicare lavoro inutile, la nuova Specula s'occupera in modo speciale della fotografia celeste. A tal fine essa è entrata nel consorzio dei pochi Osservatori astronomici disseminati in tutto l'orbe, per intraprendere il nuovo e grandioso lavoro per la formazione della carta topografica del cielo; e si è già dato ordine per la costruzione del grande e speciale istrumento richiesto, per eseguire le ricerche fotografiche coi mezzi e colle norme concertate dal Comitato internazionale permanente per la formazione della carta suddetta, residente a Parigi.

La Specula è inoltre già fornita degli istrumenti richiesti per un bene ordinato Osservatorio astronomico; tra cui una importante collezione di cronometri e cronografi, acquistata per circostanze affatto speciali e propizie.

In tal modo anche per questa parte la Specula Vaticana avrà un compito insigne, affatto nuovo, e che rimarrà memorabile nella storia dell' astronomia, siccome rimase quello, per cui essa fu stabilita fin dal suo principio.

\section{Ephemeride des Cometen 1890...(Brooks März 19). \\ (Fortsetzung zul A. N. 2970.)}

I $2^{\text {h }}$ M. Z. Berlin.

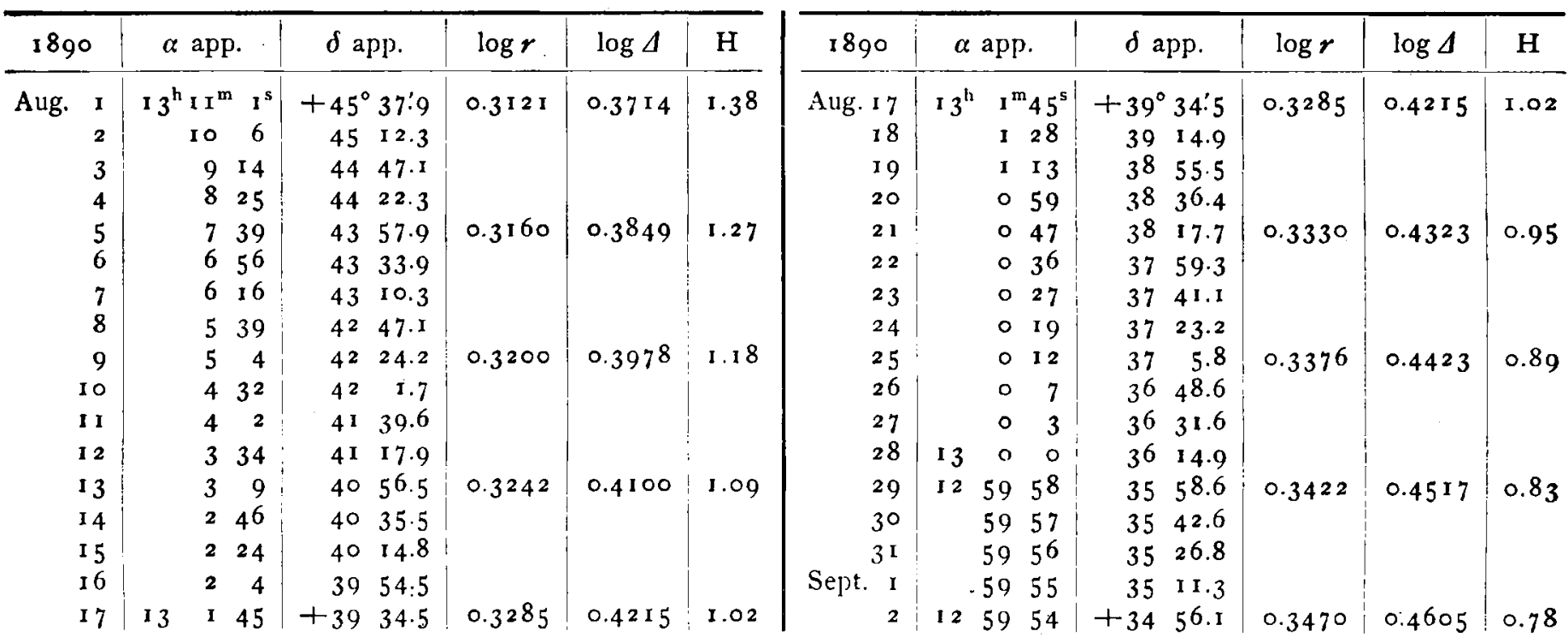

Als Einheit der Helligkeit wurde die vom 2 I. März 1890 gewählt.

Sternwarte Wien-Währing, I 890 Juni 30.

Friedrich Bidschof.

In halt zu Nr. 2979. Beobachtungen der Sonnenfinsterniss 1890 Juni $16-17$ in Strassburg, Kis Kartal, Prag, Padua, Kasan, Dresden, OGyalla Göttingen und Heidellerg. 33. - F. Porro. Osservazioni di Stelle Variabili a lungo periodo, 39. - F. Tebbutt. The Supposed Variable in RA. $=12^{\mathrm{h}} \mathrm{I} 8 \mathrm{mo}$ Decl. $=-4^{\circ} 43^{\prime}(1875.0) \cdot 4 \mathrm{I} .-S . W$. Burnlam. Measures of Doutle Stars, 4I. - G. Lorenzoni. Occultazione di Giove e dei suoi satelliti dietro la Luna ed eclisse del I. Satellite. 43. - E. E. Barnard. An unsuccessful search for Brorsen's Comet I889-90. 43. - E. Millosezich. Osservazioni della cometa I890 ... (Brooks Marzo 19). 45. - W. Luthir. Beobachtungen des Cometen 1890... (Jronks März 19) . 45. - F. Denza. Specula Vaticana. 47. - F. Bidschof. Ephemeride des Cometen $1890 \ldots$ (Brooks März I9). 47. 